Revista de Matemática: Teoría y Aplicaciones 2017 24(1) : 61-77

CIMPA - UCR ISSN: 1409-2433 (PRINT), 2215-3373 (ONLINE)

\title{
ESTABILIDAD DE SISTEMAS POR MEDIO DE POLINOMIOS HURWITZ
}

\author{
STABILITY SYSTEMS VIA HURWITZ \\ POLYNOMIALS
}

\author{
BaltaZAr AguirRe-HernÁndeZ* \\ CARlos Arturo Loredo-Villalobos ${ }^{\dagger}$ \\ Edgar CRISTIAN DÍAZ-GonZÁLEZ \\ ERIC CAMPOS-CANTÓN ${ }^{\S}$
}

Received: 17/Mar/2016; Revised: 26/Aug/2016;

Accepted: $7 / 0$ ct/2016

\footnotetext{
Revista de Matemática: Teoría y Aplicaciones is licensed under a Creative Commons Reconocimiento-NoComercial-Compartirigual 4.0 International License.

Creado a partir de la obra en $\mathrm{http}: / / \mathrm{ww}$.revistas.ucr.ac.cr/index.php/matematica

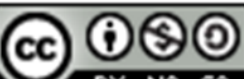

*Departamento de Matemáticas. Universidad Autónoma Metropolitana-Iztapalapa, México, D.F., México. E-Mail: bahe@ xanum.uam.mx

${ }^{\dagger}$ Universidad Autónoma Metropolitana-Iztapalapa, México, D.F. and División de Matemáticas Aplicadas, Instituto Potosino de Investigación Científica y Tecnológica, San Luis Potosí, México. E-Mail: calv@xanum.uam.mx

${ }^{\ddagger}$ Misma dirección que/Same address as: B. Aguirre-Hernández. E-Mail: edgardazgonzlez@yahoo.com.mx

${ }^{\S}$ División de Matemáticas Aplicadas. Instituto Potosino de Investigación Científica y Tecnológica, México San Luis Potosí, México. E-Mail: eric.campos@ipicyt.edu.mx
} 


\title{
Resumen
}

Para analizar la estabilidad de un sistema de ecuaciones diferenciales lineales $\dot{x}=A x$ podemos estudiar la localización de las raíces del polinomio característico $p_{A}(t)$ asociado a la matriz $A$. En este artículo presentamos diversos criterios — algebraicos y geométricos- que nos ayudan a determinar el lugar donde se encuentran las raíces sin necesidad de calcularlas en forma directa.

Palabras clave: polinomios Hurwitz; estabilidad de sistemas; criterios de estabilidad.

\begin{abstract}
To analyze the stability of a linear system of differential equations $\dot{x}=A x$ we can study the location of the roots of the characteristic polynomial $p_{A}(t)$ associated with the matrix $A$. We present various criteria - algebraic and geometric - that help us to determine where the roots are located without calculating them directly.
\end{abstract}

Keywords: Hurwitz polynomials; system stability; stability criteria.

Mathematics Subject Classification: 93C05, 93D09, 93D20.

\section{Introducción}

Consideremos a un sistema de ecuaciones diferenciales lineales $\dot{x}=A x$. El polinomio característico de un sistema lineal determina el desarrollo y las propiedades de todo el sistema. Si todos los valores propios de la matriz $A$ tienen parte real negativa entonces todas las soluciones del sistema convergen al origen cuando $t \rightarrow \infty$ y esto es llamada estabilidad asintótica de la solución trivial del sistema. Una matriz con la propiedad de que sus valores propios tienen parte real negativa se dice que es una matriz Hurwitz. Determinar la estabilidad de un sistema es uno de los problemas fundamentales de la teoría de control.

El problema de determinar la localización de las raíces de un polinomio $p(t)$ sin calcularlas en forma directa se formuló por primera vez en 1868 por J. Maxwell, quien obtuvo una solución para $n=3$. En 1877, J. Routh fue capaz de resolver de forma más general este problema. Su solución fue algorítmica, en ella se formularon condiciones explícitas para $n=4$ y $n=5$. La solución analítica del problema la obtuvo A. Hurwitz en 1895. Aunque diferentes en su forma, el algoritmo de Routh y el criterio de Hurwitz son equivalentes. 
Definición 1 (Polinomio Hurwitz) Decimos que un polinomio con coeficientes reales es Hurwitz si todas sus raíces tienen parte real negativa, i.e., están en el semiplano izquierdo del plano complejo

$$
\mathbb{C}^{-}=\{a+i b: a<0\}
$$

Ejemplo 2 Consideremos los siguiente polinomios:

1. El polinomio $p(t)=t^{2}+3 t+2$ es Hurwitz pues $p(t)=(t+2)(t+1)$, $t=-2$ y $t=-1$ son sus raices.

2. El polinomio $s(t)=t^{3}+t^{2}+t+1$ no es Hurwitz ya que $s(t)=t^{2}(t+$ $1)+t+1=\left(t^{2}+1\right)(t+1)$, sus raíces son $t=i, t=-i y t=1$.

Observación 3 No es difícil verificar lo siguiente:

i) El polinomio $p(t)=t+a_{1}$ es Hurwitz, si y sólo si, $a_{1}>0$.

ii) El polinomio $p(t)=t^{2}+a_{1} t+a_{2}$ es Hurwitz, si y sólo si, $a_{1}>0$ y $a_{2}>0$.

Ejemplo 4 A partir del apartado ii) de la observación anterior podemos decir que:

a) $p(t)=t^{2}+5 t+7$ es Hurwitz.

b) $p(t)=t^{2}+2 t-3$ no es Hurwitz.

Observación 5 Extendiendo a polinomios no mónicos la observación anterior, puede verificarse que:

i) $p(t)=b_{0} t+b_{1}$ es Hurwitz, si y sólo si, $b_{0}$ y $b_{1}$ son del mismo signo.

ii) $q(t)=a_{0} t^{2}+a_{1} t+a_{2}$ es Hurwitz, si y sólo si, $a_{0}, a_{1}$ y $a_{2}$ son del mismo signo.

Ejemplo 6 Consideremos el polinomio siguiente: $p(t)=-2 t^{2}-3 t-2$. Por el apartado ii) de la observación 5, $p(t)$ es Hurwitz. 


\subsection{Aplicación 1}

Ejemplo 7 (Oscilador armónico amortiguado) Considérese una masa $\mathrm{m}$ sujeta por un extremo a un resorte y por el otro a un amortiguador. Estiramos el resorte una cierta distancia $x$ y luego lo soltamos. El modelo para el movimiento de la masa está descrito por la siguiente ecuación

$$
m \frac{d^{2} x}{d t^{2}}+\beta \frac{d x}{d t}+k x=0
$$

donde $\beta>0$ es la constante de amortiguamiento y $k>0$ es la constante del resorte. La ecuación característica de la ecuación diferencial es

$$
p(\lambda)=m \lambda^{2}+\beta \lambda+k=0 .
$$

Cómo $m, \beta, k>0$ entonces $p(\lambda)$ es Hurwitz. Además:

1. Si $\lambda_{1}, \lambda_{2} \in \mathbb{R}^{-}$entonces

$$
x(t)=A e^{\lambda_{1} t}+B e^{\lambda_{2} t} .
$$

2. Si $\lambda_{1}=\alpha+i \beta$ y $\lambda_{2}=\alpha-i \beta$, con $\alpha<0$, entonces

$$
x(t)=A e^{\alpha t} \cos \beta t+B e^{\alpha t} \sin \beta t .
$$

Luego, cualquiera de estas soluciones $x(t)$ cumplen que $x(t) \rightarrow 0$ cuando $t \rightarrow \infty$.

\subsection{Aplicación 2}

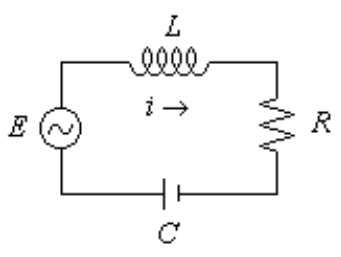

Figura 1: Circuito eléctrico RLC.

Ejemplo 8 (Circuitos eléctricos) Considere un circuito eléctrico (ver fig. 1) donde $E$ es el voltaje (medido en volts), $R$ la resistencia (medida en Ohms), 
$L$ la inductancia (medida en Henrios) y $C$ la capacitancia (medida en Faradios). El modelo para la variación del voltaje en un tiempo t está dada por la siguiente ecuación

$$
L \frac{d^{2} i}{d t^{2}}+R \frac{d i}{d t}+\frac{1}{c} i=\frac{d E}{d t} .
$$

Bajo la suposición de que E es constante, la ecuación característica es

$$
p(\lambda)=L \lambda^{2}+R \lambda+\frac{1}{c}=0 .
$$

Ya que $L, R, \frac{1}{c}>0$ se tiene que $p(\lambda)$ es Hurwitz. Por lo tanto, podemos asegurar que $i(t) \rightarrow 0$ cuando $t \rightarrow \infty$. Una explicación física de esto es que $i(t) \rightarrow 0$ cuando $t \rightarrow \infty$ debido al consumo de energía de la resistencia.

\subsection{Aplicación 3}

Ejemplo 9 (Modelo Lotka-Volterra de dos especies) El modelo está expresado por el siguiente sistema de ecuaciones diferenciales:

$$
\begin{aligned}
& \dot{x}=x(2-3 x+y), \\
& \dot{y}=y(1+2 x-3 y),
\end{aligned}
$$

donde $(0,0)$ y $(1,1)$ son los puntos críticos. La matriz Jacobiana en $(1,1)$ es dada como sigue:

$$
A=\left(\begin{array}{rr}
-3 & 1 \\
2 & -3
\end{array}\right)
$$

El polinomio característico asociado a la matriz del sistema es: $p_{A}(\lambda)=\lambda^{2}+$ $6 \lambda+7$, que puede verificarse que es Hurwitz. Por lo tanto, cerca del punto $(1,1)$ las soluciones convergen.

\section{Propiedades de los polinomios Hurwitz}

A continuación presentamos algunos resultados para polinomios Hurwitz que permiten determinar la localización de sus raíces.

Teorema 10 Si $p(t)$ es un polinomio Hurwitz de grado $n$ entonces todos sus coeficientes son del mismo signo.

Demostración. Ver [10].

El teorema anterior nos da una condición necesaria pero no suficiente como se muestra en el siguiente ejemplo. 
Ejemplo 11 Sea $p(t)=t^{3}+2 t^{2}+5 t+174$, para el polinomio $p(t)$ se tiene que:

$$
p(t)=\left(t^{2}-4 t+29\right)(t+6)=(t-2+5 i)(t-2-5 i)(t+6) .
$$

Por lo tanto, $p(t)$ no es un polinomio Hurwitz.

El siguiente resultado nos permite tener condiciones necesarias y suficientes para $n=3$.

Teorema 12 El polinomio $p(t)=t^{3}+a_{1} t^{2}+a_{2} t+a_{3}$ es Hurwitz, si y sólo si, $a_{1}, a_{2}$ y $a_{3}>0$ y $\left(a_{1} a_{2}-a_{3}\right)>0$.

Demostración. Ver [10].

Ejemplo 13 Sea $p(t)=t^{3}+3 t^{2}+3 t+1$ entonces $a_{1} a_{2}-a_{3}=8>0$. Por lo tanto, $p(t)$ es Hurwitz.

Para el caso $n=4$ se tiene el siguiente resultado.

Teorema 14 El polinomio $p(t)=t^{4}+a_{1} t^{3}+a_{2} t^{2}+a_{3} t+a_{4}$ es Hurwitz, si $y$ sólo si, $a_{1}, a_{2}, a_{3} y a_{4}>0 y\left(a_{1} a_{2} a_{3}-a_{3}^{2}-a_{1}^{2} a_{4}\right)>0$.

Demostración. Ver [10].

Ejemplo 15 Sea $p(t)=t^{4}+4 t^{3}+6 t^{2}+4 t+1$, analizando los coeficientes del polinomio se tiene que:

$$
a_{1} a_{2} a_{3}-a_{3}^{2}-a_{1}^{2} a_{4}=(4)(6)(4)-4^{2}-4^{2}(1)=64>0 .
$$

Por lo tanto, $p(t)$ es Hurwitz.

De los dos teoremas anteriores se obtiene el siguiente corolario.

\section{Corolario 16}

i) $p(t)=a_{0} t^{3}+a_{1} t^{2}+a_{2} t+a_{3}$ es Hurwitz, si y sólo si, $a_{0}, a_{1}, a_{2}, a_{3}$ son del mismo signo y $a_{1} a_{2}-a_{3} a_{0}>0$.

ii) $p(t)=a_{0} t^{4}+a_{1} t^{3}+a_{2} t^{2}+a_{3} t+a_{4}$ es Hurwitz, si y sólo si, $a_{0}, a_{1}, a_{2}, a_{3}, a_{4}$ son del mismo signo y $\left(a_{1} a_{2} a_{3}-a_{3}^{2} a_{0}-a_{1}^{2} a_{4}\right) / a_{0}>0$.

A continuación presentamos el siguiente resultado para polinomios Hurwitz. 
Teorema 17 Si $f(x)=a_{0}+a_{1} x+a_{2} x^{2}+\cdots+a_{n} x^{n}$ es Hurwitz $y \xi \in \mathbb{C}$ se tiene que:

a) Si $\operatorname{Re} \xi>0$ entonces $|f(\xi)|>|f(-\xi)|$.

b) Si $\operatorname{Re} \xi=0$ entonces $|f(\xi)|=|f(-\xi)|$.

c) Si $\operatorname{Re} \xi<0$ entonces $|f(\xi)|<|f(-\xi)|$.

Demostración. Ver [10].

Observación 18 Si $f(x)$ no cumple alguno de los incisos a), b) ó c) entonces $f(x)$ no es Hurwitz.

Ejemplo 19 Sea $f(x)=x^{3}+2 x^{2}+16 x+130$ tomando $\xi=1+5$ i tenemos:

$$
f(\xi)=(1+5 i)^{3}+3(1+5 i)^{2}+16(1+5 i)+130=0 .
$$

Luego $|f(\xi)|=0$; por otro lado

$$
f(-\xi)=(-1-5 i)^{3}+3(-1-5 i)^{2}+16(-1-5 i)+130,=116+60 i .
$$

Luego $|f(-\xi)|>0$ y no se cumple que $|f(\xi)|>|f(-\xi)|$. Por lo tanto, $f(x)$ no es Hurwitz.

Si $f(x)$ es un polinomio que cumple los incisos $a$ ), b) y $c$ ) se tiene la siguiente pregunta: ¿ $f(x)$ será Hurwitz?. En general no, para $f(x)=x^{n}(x-1)$ se cumplen todos los incisos pero $f(x)$ no es Hurwitz. Así, surge la siguiente cuestión: ¿Qué condición extra debemos agregar a los incisos $a$ ), b) y c) con la finalidad de obtener un criterio para decidir si un polinomio es Hurwitz?

\section{Criterios para determinar si un polinomio es Hurwitz}

En la búsqueda de la localización de las raíces se formuló la siguiente pregunta: ¿Cómo se puede saber si un polinomio

$$
p(t)=a_{0} t^{n}+a_{1} t^{n-1}+\cdots+a_{n-1} t+a_{n}
$$

es Hurwitz? Al final del siglo XIX el ingeniero austriaco A. Stodola, el descubridor de la teoría del vapor y las turbinas de gas, sin conocer los trabajos de Routh propuso el problema de encontrar condiciones bajo las cuales todas las raíces de 
una ecuación algebraica tuvieran parte real negativa. En 1895, A. Hurwitz basándose en los trabajos de Hermite dio una solución al problema (independiente de la dada por Routh).

Existen diversos criterios para determinar cuando un polinomio es Hurwitz. Entre estos criterios, en la actualidad clásicos, se encuentran: el criterio de Routh-Hurwitz, las condiciones de Lienard-Chipart, el teorema de Hermite-Biehler, el teorema de Leonhard-Mihailov, el test de estabilidad y el algoritmo de Routh. Cada uno de ellos, desde diferentes enfoques y con distintas herramientas matemáticas, dan condiciones necesarias y/o suficientes para responder a la pregunta de la localización de las raíces de un polinomio. A continuación presentamos brevemente algunos de estos criterios.

\subsection{Criterio de Routh-Hurwitz}

El criterio de Routh-Hurwitz es quizá el criterio más conocido y es un criterio completamente algebraico.

Teorema 20 (Routh-Hurwitz) Dado un polinomio con coeficientes reales $f(t)=b_{0} t^{n}+b_{1} t^{n-1}+\cdots+b_{n-1} t+b_{n}$ definimos la matriz Hurwitz asociada a este polinomio de la siguiente forma:

$$
\left(\begin{array}{ccccc}
b_{1} & b_{0} & 0 & \cdots & 0 \\
b_{3} & b_{2} & b_{1} & \cdots & 0 \\
\vdots & \vdots & \vdots & \vdots & \vdots \\
b_{2 n-3} & b_{2 n-4} & b_{2 n-5} & \cdots & b_{n-2} \\
b_{2 n-1} & b_{2 n-2} & b_{2 n-3} & \cdots & b_{n}
\end{array}\right)
$$

donde $b_{k}=0$ si $k>n$. Para que tal polinomio tenga todas sus raíces con parte real negativa es necesario y suficiente que se satisfaga lo siguiente:

$$
\begin{gathered}
b_{0} \Delta_{1}>0, \Delta_{2}>0, b_{0} \Delta_{3}>0, \\
\Delta_{4}>0, \ldots\left\{\begin{array}{cc}
b_{0} \Delta_{n}>0, & \text { si } n \text { es impar } \\
\Delta_{n}>0, & \text { si } n \text { es par. }
\end{array}\right.
\end{gathered}
$$

donde los $\Delta_{i}$ son los menores principales de la matriz Hurwitz, es decir:

$$
\Delta_{1}=\left|b_{1}\right|, \Delta_{2}=\left|\begin{array}{ll}
b_{1} & b_{0} \\
b_{3} & b_{2}
\end{array}\right|, \ldots
$$

En caso de que $b_{0}=1$ la condición simplemente dice que los menores principales deben ser positivos, es decir

$$
\Delta_{1}>0, \Delta_{2}>0, \Delta_{3}>0, \ldots, \Delta_{n}>0 .
$$


Demostración. Ver [3], [10] y [1].

Ejemplo 21 Considérese $f(t)=t^{5}+7 t^{4}+19 t^{3}+25 t^{2}+16 t+4$, tomando $b_{0}=1, b_{1}=7, b_{2}=19, b_{3}=25, b_{4}=16$ y $b_{5}=4$, construimos la matriz Hurwitz de $f(t)$ :

$$
H(f)=\left(\begin{array}{ccccc}
7 & 25 & 4 & 0 & 0 \\
1 & 19 & 16 & 0 & 0 \\
0 & 7 & 25 & 4 & 0 \\
0 & 1 & 19 & 16 & 0 \\
0 & 0 & 7 & 25 & 4
\end{array}\right)
$$

Luego

$$
\begin{aligned}
& \Delta_{1}=7>0, \quad \Delta_{2}=7(19)-25>0, \\
& \Delta_{3}=25[7(19)-25]-7[7(16)-4]=2700-756>0, \\
& \Delta_{4}=16[2700]-19[4(7(19)-25)-7(0)]+[4(7(16)-4)-25(0)]>0 \\
& \Delta_{5}=4 \Delta_{4}>0 .
\end{aligned}
$$

Por el teorema 20, $f(t)$ es Hurwitz.

Ejemplo 22 Determinar si $f(t)=t^{3}+3 t^{2}+2 t+8$ es Hurwitz. Hacemos $b_{0}=1, b_{1}=3, b_{2}=2 y b_{3}=8$. Construimos la matriz Hurwitz de $f(t)$ :

$$
H(f)=\left(\begin{array}{lll}
3 & 8 & 0 \\
1 & 2 & 0 \\
0 & 3 & 8
\end{array}\right) .
$$

No es difícil verificar que $\Delta_{1}>0$ y $\Delta_{2}<0$, por lo tanto según el teorema 20, $f(t)$ no es Hurwitz.

\subsection{Condiciones de Lienard-Chipart}

Teorema 23 (Lienard-Chipart) El polinomio $f(t)$ con $b_{0}>0$ es Hurwitz, si y sólo si, satisface alguna de las siguientes condiciones:

$$
\begin{array}{ll}
\text { 1. } b_{n}>0, b_{n-2}>0, b_{n-4} \ldots ; & \Delta_{1}>0, \Delta_{3}>0, \Delta_{5}>0 \ldots, \\
\text { 2. } b_{n}>0, b_{n-2}>0, b_{n-4} \ldots ; & \Delta_{2}>0, \Delta_{4}>0, \Delta_{6}>0 \ldots, \\
\text { 3. } b_{n}>0, b_{n-1}>0, b_{n-3} \ldots ; & \Delta_{1}>0, \Delta_{3}>0, \Delta_{5}>0 \ldots, \\
\text { 4. } b_{n}>0, b_{n-1}>0, b_{n-3} \ldots ; & \Delta_{2}>0, \Delta_{4}>0, \Delta_{6}>0 \ldots .
\end{array}
$$


Demostración. Ver [6].

Ejemplo 24 Verificar si $f(t)=t^{5}+10 t^{4}+40 t^{3}+80 t^{2}+80 t+1$ es Hurwitz. Construimos la matriz Hurwitz de $f(t)$. Así, tenemos que:

$$
H(f)=\left(\begin{array}{ccccc}
10 & 80 & 1 & 0 & 0 \\
1 & 40 & 80 & 0 & 0 \\
0 & 10 & 80 & 1 & 0 \\
0 & 1 & 40 & 80 & 0 \\
0 & 0 & 10 & 80 & 1
\end{array}\right)
$$

Donde $b_{5}=1, b_{3}=80, b_{1}=10$ y $\Delta_{1}=10>0$, además:

$$
\begin{aligned}
& \Delta_{3}=-10[800-1]+80[400-80]=-7990+25600>0 \\
& \Delta_{5}=80 \Delta_{3}-\operatorname{det}\left(\begin{array}{ccc}
10 & 80 & 10 \\
1 & 40 & 80 \\
0 & 1 & 40
\end{array}\right)=80 \Delta_{3}-12001>0 .
\end{aligned}
$$

Por el inciso 1) del teorema $23 f(t)$ es Hurwitz.

Del criterio anterior tenemos la siguiente pregunta abierta: ¿será o no posible mejorar las condiciones de Lienard-Chipart?, es decir, ¿el número de desigualdades por calcular podrá reducirse?

\subsection{Teorema de Hermite-Biehler}

A continuación presentamos este criterio el cual es de tipo geométrico. Consideremos el polinomio $p(t)$ con coeficientes reales: $p(t)=a_{0}+a_{1} t+a_{2} t^{2}+\cdots+$ $a_{n} t^{n}$. Podemos escribir $p(t)$ de la siguiente forma:

$$
p(t)=\left(a_{0}+a_{2} t^{2}+a_{4} t^{4}+\cdots\right)+t\left(a_{1}+a_{3} t^{2}+a_{5} t^{4}+\cdots\right)
$$

evaluando en $i \omega$ tenemos que:

$$
p(i \omega)=\left(a_{0}-a_{2} \omega^{2}+a_{4} \omega^{4}-\cdots\right)+i \omega\left(a_{1}-a_{3} \omega^{2}+a_{5} \omega^{4}-\cdots\right) .
$$

Definimos los polinomios siguientes:

$$
\begin{aligned}
p^{\text {par }}(t) & :=a_{0}+a_{2} t^{2}+a_{4} t^{4}+\cdots, \\
p^{\text {impar }}(t) & :=a_{1} t+a_{3} t^{3}+a_{5} t^{5}+\cdots, \\
p^{p}(\omega) & :=p^{\text {par }}(j \omega)=a_{0}-a_{2} \omega^{2}+a_{4} \omega^{4}-\cdots, \\
p^{i m}(\omega) & :=\left(p^{\text {impar }}(j \omega)\right) / j \omega=a_{1}-a_{3} \omega^{2}+a_{5} \omega^{4}-\cdots,
\end{aligned}
$$


donde $p^{p}(\omega)$ y $p^{i m}(\omega)$ son ambos polinomios en $\omega^{2}$ y como consecuencia, cada conjunto de raíces será siempre simétrico con respecto al origen del plano complejo.

Supongamos ahora que el grado del polinomio $p(t)$ es par, $n=2 m, m>0$. En este caso, tenemos lo siguiente:

$$
\begin{aligned}
p^{p}(\omega) & =a_{0}-a_{2} \omega^{2}+a_{4} \omega^{4}-\cdots+(-1)^{m} a_{2 m} \omega^{2 m}, \\
p^{i m}(\omega) & =a_{1}-a_{3} \omega^{2}+a_{5} \omega^{4}-\cdots+(-1)^{m-1} a_{2 m-1} \omega^{2 m-2} .
\end{aligned}
$$

Definición 25 Un polinomio real $p(t)$ satisface la propiedad de la alternancia, si:

a) $a_{2 m}$ y $a_{2 m-1}$ tienen el mismo signo.

b) Todas las raíces de $p^{p}(\omega)$ y $p^{i m}(\omega)$ son reales, distintas y además las $m$ raíces positivas de $p^{p}(\omega)$ y las $m-1$ raíces positivas de $p^{i m}(\omega)$ se van alternando, es decir:

$$
0<\omega_{e, 1}<\omega_{o, 1}<\omega_{e, 2}<\omega_{o, 2}<\cdots<\omega_{e, m-1}<\omega_{o, m-1}<\omega_{e, m}
$$

Si por el contrario, el grado de $p(t)$ es impar entonces $n=2 m+1, m \geq 0 \mathrm{y}$

$$
\begin{aligned}
p^{p}(\omega) & =a_{0}-a_{2} \omega^{2}+a_{4} \omega^{4}-\cdots+(-1)^{m} a_{2 m} \omega^{2 m}, \\
p^{i m}(\omega) & =a_{1}-a_{3} \omega^{2}+a_{5} \omega^{4}-\cdots+(-1)^{m} a_{2 m+1} \omega^{2 m} .
\end{aligned}
$$

La definición de la propiedad de alternancia es modificada:

a) $a_{2 m+1}$ y $a_{2 m}$ tienen el mismo signo.

b) Todas las raíces de $p^{p}(\omega)$ y $p^{i m}(\omega)$ son reales, distintas y las $m$ raíces positivas de $p^{p}(\omega)$ juntamente con las $m$ raíces positivas de $p^{i m}(\omega)$ se van alternando, es decir:

$$
0<\omega_{e, 1}<\omega_{o, 1}<\cdots<\omega_{e, m-1}<\omega_{o, m-1}<\omega_{e, m}<\omega_{o, m}
$$

Ahora podemos enunciar el siguiente criterio de estabilidad.

Teorema 26 (Hermite-Biehler o de la Alternancia) Un polinomio real $p(t)$ es Hurwitz, si y sólo si, satisface la propiedad de la alternancia.

Demostración. Ver [3], [4] y [8]. 
Ejemplo 27 Sea

$$
\begin{aligned}
p(t)= & 147+574 t+1363 t^{2}+2103 t^{3}+2402 t^{4}+2015 t^{5} \\
& +1293 t^{6}+614 t^{7}+221 t^{8}+57 t^{9}+10 t^{10}+t^{11}
\end{aligned}
$$

entonces

$$
p(j \omega)=p^{p}(\omega)+j \omega p^{i m}(\omega)
$$

con

$$
\begin{aligned}
p^{p}(\omega)= & 147-1363 \omega^{2}+2402 \omega^{4}-1293 \omega^{6} \\
& +221 \omega^{8}-10 \omega^{10} \\
p^{i m}(\omega)= & 574-2103 \omega^{2}+2015 \omega^{4}-614 \omega^{6} \\
& +57 \omega^{8}-\omega^{10}
\end{aligned}
$$

Las gráficas de $p^{p}(\omega)$ y $p^{i m}(\omega)$ se muestran en la figura 2.

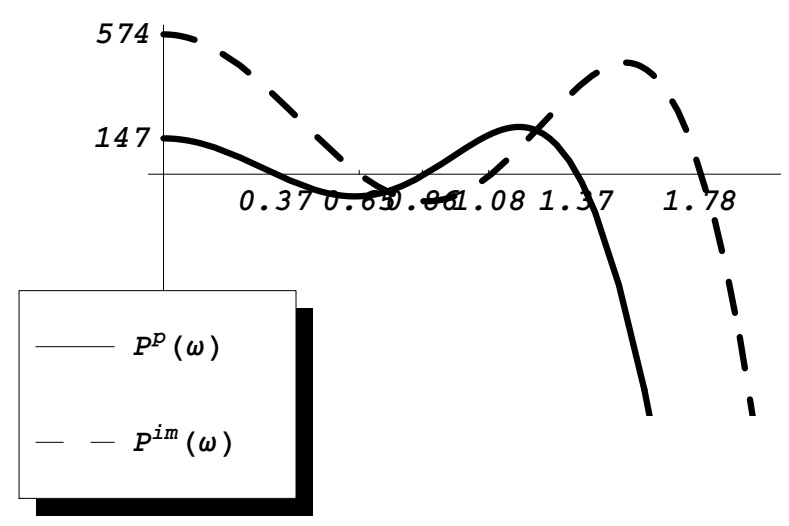

Figura 2: Teorema de la alternancia aplicado a polinomios Hurwitz( Ejem. 27).

\subsection{Teorema de Leonhard-Mihailov}

A continuación se presenta un criterio debido a V. V. Mihailov (a veces también llamado criterio de Leonhard) y puede considerarse también un criterio de tipo geométrico. 
Teorema 28 (Leonhard-Mihailov) Un polinomio con coeficientes reales $p(t)=$ $a_{0} t^{n}+a_{1} t^{n-1}+\cdots+a_{n}$ es Hurwitz, si y sólo si, el argumento de $p(i \omega)$, $\arg (p(i \omega))$, es una función de $\omega$ continua y estrictamente creciente sobre $(-\infty, \infty)$. Además, el incremento neto del argumento de $-\infty$ a $\infty$ es $n \pi$, es decir, $\arg [P(+i \infty)]-\arg [P(-i \infty)]=n \pi$.

Demostración. Ver [10].

La curva que describe $p(i \omega)$ en el plano complejo, cuando $\omega$ recorre el intervalo $[0,+\infty)$ se llama hodógrafo de Mihailov o diagrama de respuesta de Leonhard.

Ejemplo 29 Consideremos el polinomio $p(t)=t^{3}+3 t^{2}+3$, calculamos $p(i \omega)$ :

$$
\begin{aligned}
p(i \omega) & =(i \omega)^{3}+3(i \omega)^{2}+(i \omega)+3, \\
& =3-3 \omega^{2}+i \omega\left(1-\omega^{2}\right),
\end{aligned}
$$

entonces la fase de $p(i \omega)$ es:

$$
\begin{aligned}
\arg p(i \omega) & =\arctan \frac{\omega\left(1-\omega^{2}\right)}{3-3 \omega^{2}} \\
& =\arctan \frac{\omega\left(1-\omega^{2}\right)}{3\left(1-\omega^{2}\right)} \\
& =\arctan \frac{\omega}{3}
\end{aligned}
$$

Luego

$$
\begin{aligned}
& \lim _{\omega \rightarrow+\infty} \arg p(i \omega)=\lim _{\omega \rightarrow+\infty} \arctan \frac{\omega}{3}=\frac{\pi}{2}, \\
& \lim _{\omega \rightarrow-\infty} \arg p(i \omega)=\lim _{\omega \rightarrow-\infty} \arctan \frac{\omega}{3}=-\frac{\pi}{2} .
\end{aligned}
$$

Por lo tanto:

$$
\arg [p(+i \infty)]-\arg [p(-i \infty)]=\pi \neq 3 \pi
$$

Así, por el teorema 28, $p(t)$ no es Hurwitz.

Si además la curva $p(i \omega)$ no se intersecta entonces se tienen las siguientes cuestiones: ¿cómo serán las raíces de $p(t)$ ?, ¿cuáles serían las implicaciones prácticas? 


\subsection{Test de estabilidad}

Definición 30 Dado el polinomio $P(t)=a_{n} t^{n}+a_{n-1} t^{n-1}+\ldots+a_{1} t+a_{0}$, si $a_{n-1} \neq 0$ definimos

$$
\begin{aligned}
Q(t)= & a_{n-1} t^{n-1}+\left(a_{n-2}-\frac{a_{n}}{a_{n-1}} a_{n-3}\right) t^{n-2}+ \\
& +a_{n-3} t^{n-3}+\left(a_{n-4}-\frac{a_{n}}{a_{n-1}} a_{n-5}\right) t^{n-4}+\ldots \text { (*). }^{*}
\end{aligned}
$$

Teorema 31 (Test de estabilidad) Si $P(t)$ tiene todos sus coeficientes positivos; $P(t)$ es Hurwitz, si y sólo si, $Q(t)$ es Hurwitz.

Demostración. Ver [3] y [10].

El teorema anterior muestra cómo verificar si un polinomio $P(t)$ es Hurwitz por medio de la reducción sucesiva de su grado.

Ejemplo 32 Verificar si el polinomio $q(t)=t^{5}+5 t^{4}+10 t^{3}+10 t^{2}+5 t+1$ es Hurwitz. Hacemos

$$
P^{(0)}(t)=t^{5}+5 t^{4}+10 t^{3}+10 t^{2}+5 t+1 .
$$

Luego construimos $P^{(1)}(t)$ :

$$
P^{(1)}(t)=5 t^{4}+\left(10-\frac{1}{5} 10\right) t^{3}+10 t^{2}+\left(5-\frac{1}{5} 1\right) t+1=5 t^{4}+8 t^{3}+10 t^{2}+\frac{24}{5} t+1 .
$$

Los coeficientes de $P^{(1)}(t)$ son todos positivos entonces construimos $P^{(2)}(t)$ :

$$
P^{(2)}(t)=8 t^{3}+\left(10-\frac{5}{8}\left(\frac{24}{5}\right)\right) t^{2}+\frac{24}{5} t+\left(1-\frac{5}{8} 0\right)=8 t^{3}+7 t^{2}+\frac{24}{5} t+1 .
$$

Vemos que $P^{(2)}(t)$ de nuevo tiene sus coeficientes positivos entonces construi$\operatorname{mos} P^{(3)}(t)$ :

$$
P^{(3)}(t)=7 t^{2}+\left(\frac{24}{5}-\frac{8}{7}(1)\right) t+17 t^{2}+\frac{128}{75} t+1 .
$$

Como $P^{(3)}(t)$ es de grado 2 y todos sus coeficientes son positivos entonces por el teorema 31 podemos concluir que $q(t)$ es Hurwitz. 


\subsection{Algoritmo de Routh}

Este criterio de estabilidad hace uso de un arreglo que nos ayuda a determinar la localización de las raíces de un polinomio real $p(t)$.

Consideremos la siguiente notación para el polinomio $p(t)$ :

$$
p(t)=a_{0} t^{n}+b_{0} t^{n-1}+a_{1} t^{n-2}+b_{1} t^{n-3}+\cdots\left(a_{0} \neq 0\right) .
$$

Así, construimos los coeficientes $c_{0}, c_{1}, \ldots$ mediante el siguiente algoritmo:

$$
c_{0}=a_{1}-\frac{a_{0}}{b_{0}} b_{1}=\frac{b_{0} a_{1}-a_{0} b_{1}}{b_{0}}, \quad c_{1}=a_{2}-\frac{a_{0}}{b_{0}} b_{2}=\frac{b_{0} a_{2}-a_{0} b_{2}}{b_{0}}, \ldots
$$

De forma similar, tenemos que:

$$
d_{0}=b_{1}-\frac{b_{0}}{c_{0}} c_{1}=\frac{c_{0} b_{1}-b_{0} c_{1}}{c_{0}}, \quad d_{1}=b_{2}-\frac{b_{0}}{c_{0}} c_{2}=\frac{c_{0} b_{2}-b_{0} c_{2}}{c_{0}}, \ldots
$$

Los coeficientes siguiente se determinan de la misma manera. Definimos para los coeficientes del polinomio $f(t)$ el arreglo de Routh de la forma siguiente:

$\begin{array}{cccc}a_{0} & a_{1} & a_{2} & \cdots \\ b_{0} & b_{1} & b_{2} & \cdots \\ c_{0} & c_{1} & c_{2} & \cdots \\ d_{0} & d_{1} & d_{2} & \cdots \\ \cdots & \cdots & \cdots & \cdots\end{array}$

Así, tenemos el siguiente teorema de Routh.

Teorema 33 (Routh) El número de raíces del polinomio real $f(t)$ en el semiplano derecho del plano complejo es igual al número de variaciones de signo en la primera columna del arreglo de Routh.

Demostración. Ver [6] y [10]. a Para el caso de estabilidad tenemos el siguiente resultado.

Teorema 34 (Algoritmo de Routh) Todas las raíces del polinomio real $f(t)$ tienen parte real negativa, si y sólo si, mediante la utilización del algoritmo de Routh todos los elementos de la primera columna del arreglo de Routh son diferentes de cero y tienen el mismo signo.

Demostración. Ver [6] y [10]. 
Ejemplo 35 Determinar si la siguiente ecuación característica representa un sistema estable:

$$
f(t)=t^{3}+4 t^{2}+8 t+12=0 .
$$

El arreglo de Routh para este sistema es:

$\begin{array}{cc}1 & 8 \\ 4 & 12 \\ 5 & 0 \\ 12 & \end{array}$

Ya que no hay cambios de signo en la primera columna, por el teorema 34, todas las raíces de la ecuación característica tienen partes reales negativas y el sistema es estable.

\section{Conclusiones}

En este artículo hemos presentado algunos resultados clásicos que nos ayudan a determinar la localización de las raíces de polinomios reales mediante el análisis de sus coeficients. Esto nos permite estudiar la estabilidad de un sistema de ecuaciones diferenciales lineales invariantes, $\dot{x}=A x$, por medio del polinomio característico asociado a la matriz $A$. De los criterios aquí expuestos, el criterio de Routh- Hurwitz es quizá el más utilizado debido a la simplicidad de los cálculos que deben realizarse y a que utiliza nociones elementales del álgebra lineal.

\section{Referencias}

[1] Anagnost, J.J. Desoer, C.A. (1991). "An elementary proof of the RouthHurwitz stability criterion”, Circuits, Systems and Signal Processing 10(1): 101-114.

[2] Barmish, B.R. (1994) New Tools for Robustness of Linear Systems. Macmillan Publishing Co., New York.

[3] Bhattacharayya, S.P.; Chapellat, H.; Keel, L.H. (1995) Robust Control. The Parametric Approach. Prentice-Hall, Upper Saddle River NJ.

[4] Díaz González, E.C. (2010) El Teorema de Hermite-Biehler. Tesis de Maestría, UAM-Iztapalapa, México, D. F. 
[5] Frank, E. (1946) On the zeros of polynomials with complex coefficients. Bull. Amer. Math. Soc. 52(2): 144-157.

[6] Gantmacher, F.R. (1959) The Theory of Matrices. Chelsea Publishing Co., New York.

[7] Guillemin, E.A. (1949) The Mathematics of Circuit Analysis. John Wiley and Sons, New York.

[8] Holtz, O. (2003) Hermite-Biehler, Routh-Hurwitz and total positivity. Linear Algebra and its Applications 372(1): 105-110.

[9] Hirsch, M.W.; Smale, S. (1974) Differential Equations, Dynamical Systems and Linear Algebra. Academic Press, New York.

[10] Loredo, C.A. (2004) "Criterios para determinar si un polinomio es polinomio Hurwitz", Reporte de los Seminarios de Investigación I y II. UAMIztapalapa, México, D.F.

[11] Lyapunov, A.M. (1992) The General Problem of the Stability of Motion. Translated by A.T. Fuller from E. Davaux's French translation (1907) of the original Russian dissertation (1892), Taylor and Francis, London. 
